\title{
Assessment of Technetium Leachability in Cement-Stabilized Basin 43 Groundwater Brine
}

\author{
G. A. Cooke, J. B. Duncan, L. L. Lockrem \\ CH2M HILL Hanford Group, Inc. \\ Richland, WA 99352 \\ U.S. Department of Energy Contract DE-AC27-99RL14047

$\begin{array}{lll}\text { EDT/ECN: } & \text { DRF } & \text { UC: } \\ \text { Cost Center: } & 74 \mathrm{~A} 10 & \text { Charge Code: } \\ \text { B\&R Code: } & & \text { Total Pages: } 15\end{array}$

Key Words: Effluent Treatment Facility, Basin 43, technetium, radionuclide, simulant, brine, TCLP, ANSI, assessment, cementitious, results

Abstract: This report is an initial report on the laboratory effort executed under RPP-PLAN-33338, Test Plan for the Assessment of Technetium Leachability in Cement-Stabilized Basin 43 Groundwater Brine. This report delineates preliminary data obtained under subcontract 21065 , release 30 , from the RJ Lee Group, Inc., Center for Laboratory Sciences. The report is predicated on CLS RPT-816, Draft Report: Assessment of Technetium Leachability in Cement Stabilized Basin 43 Groundwater Brine. This document will be revised on receipt of the final RJ Lee Group, Inc., Center for Laboratory Sciences report, which will contain data subjected to quality control and quality assurance criteria.

TRADEMARK DISCLAIMER. Reference herein to any specific commercial product, process, or service by trade name, trademark, manufacturer, or otherwise, does not necessarily constitute or imply its endorsement, recommendation, or favoring by the United States Government or any agency thereof or its contractors or subcontractors.

Printed in the United States of America. To obtain copies of this document, contact: Document Control Services, P.O. Box 950, Mailstop H6-08, Richland WA 99352, Phone (509) 372-2420; Fax (509) 376-4989.
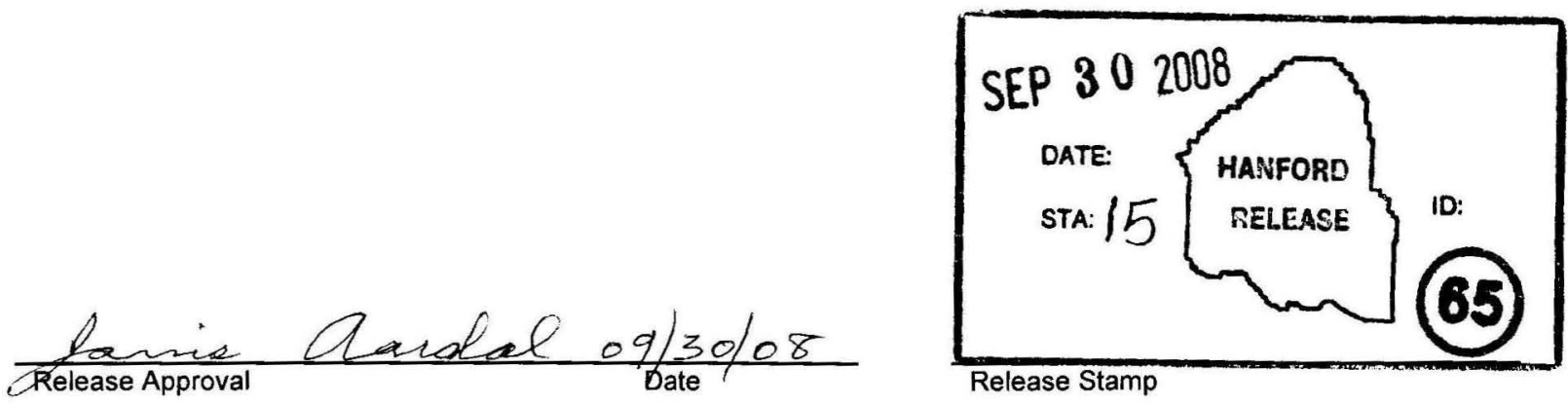

\section{Approved For Public Release}


RPP-RPT-39195

Revision 0

\title{
Assessment of Technetium Leachability in Cement-Stabilized Basin 43 Groundwater Brine
}

\author{
J. B. Duncan \\ G. A. Cooke \\ L. L. Lockrem \\ CH2M HILL Hanford Group, Inc.
}

M. D. Guthrie

Fluor Hanford

Date Published

September 2008

\section{CH2MHILL}

Hanford Group, Inc.

Prepared for the U.S. Department of Energy

Office of River Protection

Contract No. DE-AC27-99RL14047 


\section{CONTENTS}

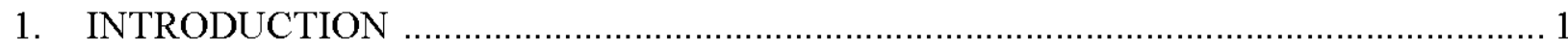

2. MATERIALS AND METHODS …................................................................... 1

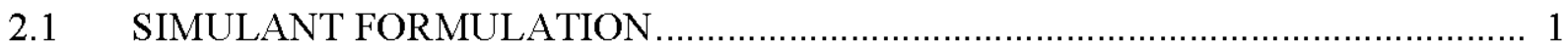

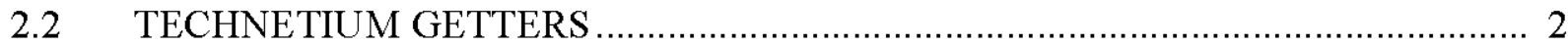

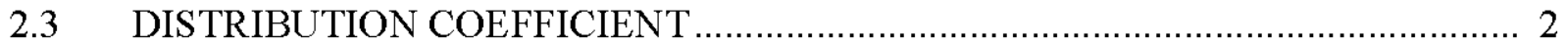

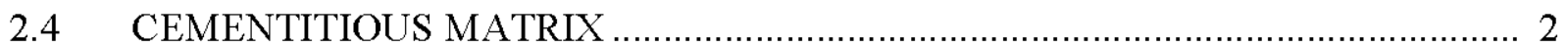

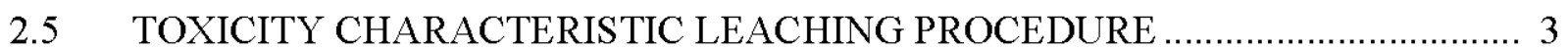

2.6 AMERICAN NATIONAL STANDARDS INSTITUTE LEACH PROCEDURE ....... 4

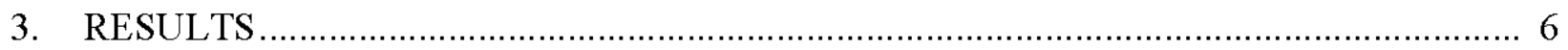

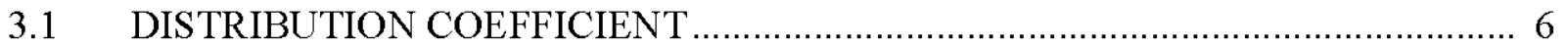

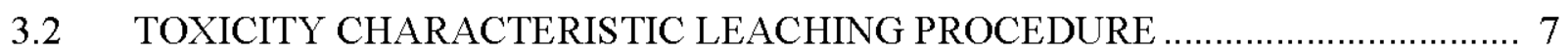

3.3 AMERICAN NATIONAL STANDARDS INSTITUTE LEACH PROCEDURE ....... 8

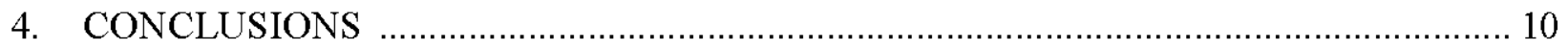

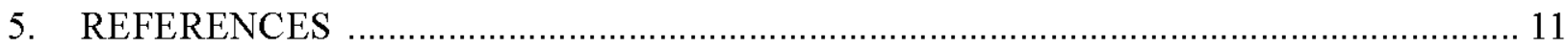

\section{List of Figures}

Figure 1. Flowsheet for the Determination of the Technetium Distribution Coefficient $\left(\mathrm{K}_{\mathrm{d}}\right)$..... 3

\section{List of Tables}

Table 1. Simulant Formulation for Basin 43 Evaporated Groundwater. ............................... 1

Table 2. Candidate Technetium Getters.................................................................................. 2

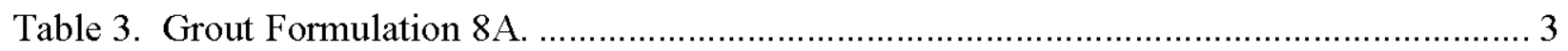

Table 4. Casting Matrix for TCLP and ANSI Procedures. (2 sheets) ...................................... 4

Table 5. ANSI Leach Schedule................................................................................ 6

Table 6. Preliminary Results Distribution Coefficients (ORP adjusted are highlighted)............ 6

Table 7. Preliminary Results Percent Recovery, (ORP adjusted are highlighted)...................... 7

Table 8. Preliminary Results of the TCLP (ORP adjusted are checked)................................... 7

Table 9. ANSI/ANS 16.1 ICP-MS Preliminary Data. (3 sheets) ......................................... 8

\section{Acronyms}

ANSI American National Standards Institute

EPA U.S. Environmental Protection Agency

ICP-MS inductively coupled plasma-mass spectrometer

ORP oxidation-reduction potential

TCLP Toxicity Characteristic Leaching Procedure 


\section{INTRODUCTION}

This report is an initial report on the laboratory effort executed under RPP-PLAN-33338, Test Plan for the Assessment of Technetium Leachability in Cement-Stabilized Basin 43 Groundwater Brine. This report delineates preliminary data obtained under subcontract 21065 , release 30 , from the RJ Lee Group, Inc., Center for Laboratory Sciences. The report is predicated on CLS-RPT-816, Draft Report: Assessment of Technetium Leachability in Cement Stabilized Basin 43 Groundwater Brine. This document will be revised on receipt of the final RJ Lee Group, Inc., Center for Laboratory Sciences report, which will contain data subjected to quality control and quality assurance criteria.

\section{MATERIALS AND METHODS}

This section describes the activities around the Basin 43 Groundwater simulant (referred hereafter as simulant), testing of technetium getters, distribution coefficients $\left(\mathrm{K}_{\mathrm{d}}\right)$, cement matrix, Toxicity Characteristic Leaching Procedure (TCLP), and the American National Standards Institute Leach Procedure (ANSI Leach). The preliminary results are reported in Chapter 3.

\subsection{SIMULANT FORMULATION}

The simulant used is shown in Table 1. The simulant is a concentrated brine consisting of approximately $25 \mathrm{wt} \%$ salts.

Table 1. Simulant Formulation for Basin 43 Evaporated Groundwater.

\begin{tabular}{|c|c|}
\hline Reagent & Gram/Liter \\
\hline $\mathrm{NaNO}_{3}$ & 140.7948 \\
\hline $\mathrm{MgSO}_{4}$ & 19.6177 \\
\hline $\mathrm{AlCl}_{3}$ & 0.1337 \\
\hline $\mathrm{KCl}$ & 2.7886 \\
\hline $\mathrm{BaCl}_{2}$ & 0.0235 \\
\hline $\mathrm{NaF}$ & 1.4126 \\
\hline $\mathrm{CaCl}_{2}$ & 7.9349 \\
\hline $\mathrm{Na}_{2} \mathrm{CrO}_{4}$ & 0.1424 \\
\hline $\mathrm{Ca}(\mathrm{OH})_{2}$ & 5.5384 \\
\hline $\mathrm{Ca}\left(\mathrm{NO}_{3}\right)_{2}$ & 42.3001 \\
\hline $\mathrm{NaHCO}_{3}$ & 37.8555 \\
\hline Total & 258.5422 \\
\hline
\end{tabular}

The simulant used was formulated to match the anticipated Basin 43 waste from the Effluent Treatment Facility. The simulant is based on data obtained from wells 299-W11-45 and 299-W11-46. 


\subsection{TECHNETIUM GETTERS}

The technetium getters initially chosen for the effort were identified in RPP-RPT-26742, Hanford Containerized Cast Stone Facility Task 1 - Process Testing and Development Final Test Report. The initial technetium getters are presented in Table 2.

Table 2. Candidate Technetium Getters.

\begin{tabular}{|l|l|}
\hline \multicolumn{1}{|c|}{ Material } & \multicolumn{1}{c|}{ Source } \\
\hline Bone black & Ebonex \\
\hline Fish bone & UFA ventures \\
\hline Ferrous sulphate & Fisher Scientific \\
\hline Zero valent iron & Fisher Scientific \\
\hline Sodium metabisulfite & Effluent Treatment Facility \\
\hline
\end{tabular}

During the progression of the laboratory effort, other getters were identified. The first of which was iron (III) phosphate, which was tested immediately after the candidates in Table 2 . The sodium metabisulfite was discarded due to the deleterious effect on the chrome passivating layer in 304 and 316L stainless steel. The revision will also report on those getters that were identified later into the laboratory effort, and experimental data is not yet available as of this report.

\subsection{DISTRIBUTION COEFFICIENT}

Distribution coefficients $\left(\mathrm{K}_{\mathrm{d}}\right)$ were calculated from data gathered using the following experiment:

To a simulant solution of known $\mathrm{TcO}_{4}{ }^{-}$concentration (currently estimated to be $92.17 \mathrm{ppm} \mathrm{Tc}^{-7}$ or $1.5 \mathrm{E}-03 \mathrm{Ci} / \mathrm{L}$ ), the following conditions applied for each material listed in Table 2 (Figure 1):

- Solution 1 - No oxidation-reduction potential (ORP) adjust (blank)

- Solution 2 - ORP adjust (to $-400 \mathrm{mV}$ ) + no getter

- Solution 3 - ORP adjust (to $-400 \mathrm{mV}$ ) + getter

- Solution 4 - Getter no ORP adjust

\subsection{CEMENTITIOUS MATRIX}

After the distribution coefficient tests, the samples were cast in the grout formulation shown in Table 3. This formulation was modified from formulation 8A (referenced in RPP-PLAN-33338) to reduce excess bleedwater. The cement samples cured for a 28 -day period before final leach testing. The castings were cured at approximately $130^{\circ} \mathrm{F}$ for a minimum of 24 hours to simulate temperatures that would be encountered if cast as a large monolith. 
Table 3. Grout Formulation 8A.

\begin{tabular}{|l|c|}
\hline \multicolumn{1}{|c|}{ Material } & Amount \\
\hline Brine:Solids ratio & $1.1: 1$ \\
\hline Basin 43 waste & $110 \mathrm{~g}$ \\
\hline Portland cement & $66.6 \mathrm{~g}$ \\
\hline Slag & $33.3 \mathrm{~g}$ \\
\hline
\end{tabular}

Figure 1. Flowsheet for the Determination of the Technetium Distribution Coefficient $\left(K_{d}\right)$.

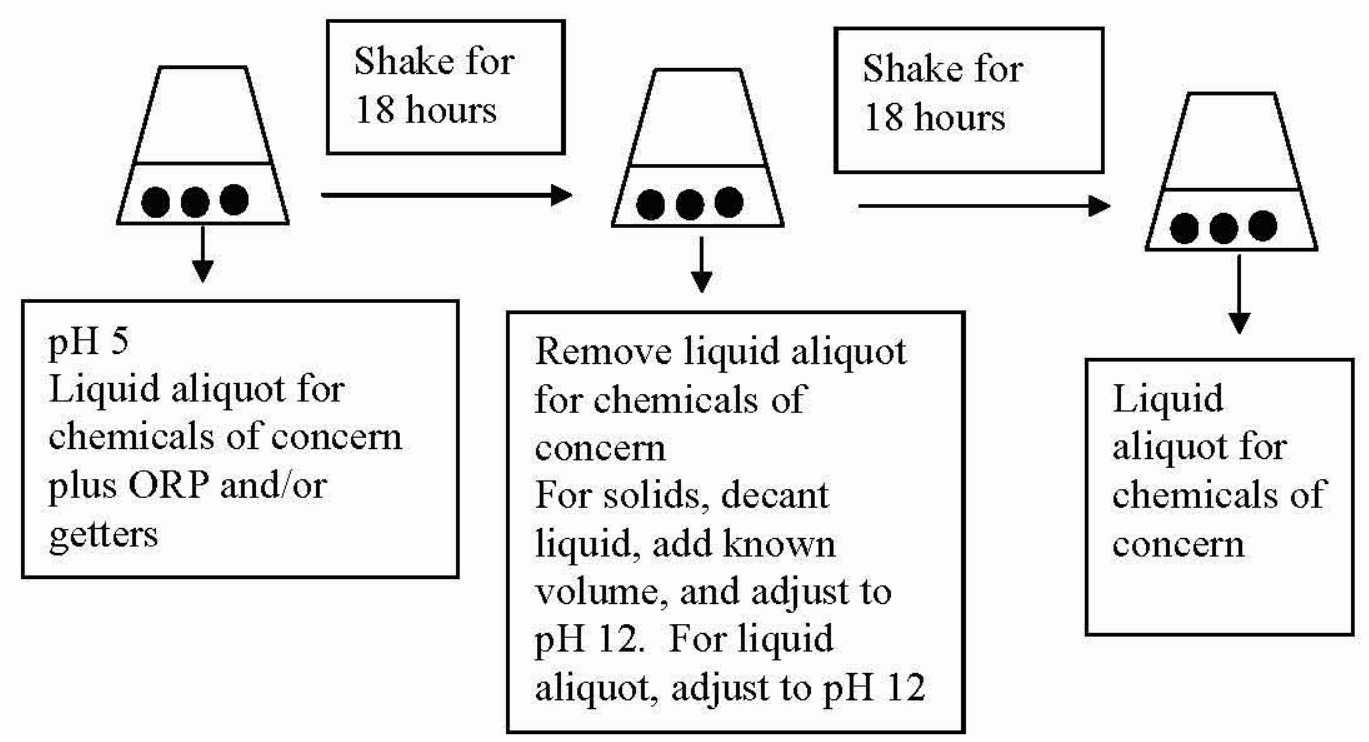

\subsection{TOXICITY CHARACTERISTIC LEACHING PROCEDURE}

U.S. Environmental Protection Agency (EPA) SW-846 Method 1311, "Toxicity Characteristic Leaching Procedure," was carried out on the cured samples. The resultant leacheate was analyzed for technetium using an inductively coupled plasma-mass spectrometer (ICP-MS) PerkinElmer ${ }^{1}$ ELAN DRC-e.

At the conclusion of the 28-day cure period the monoliths identified for TCLP analysis were crushed in their casting vessels to the necessary grain size. Each monolith yielded $\sim 40$ to $56 \mathrm{~g}$ of sample, which was added to $\sim 800$ to $1000 \mathrm{~g}$ of TCLP extraction fluid 2 (glacial acetic acid at $\mathrm{pH}$ of 2.88), and then subjected to continuous rotation at $30 \pm 2 \mathrm{rpm}$ for $18 \pm 2$ hours. The leached portions continued to be subjected to the acetic acid environment for an additional 24 hours prior to the removal of aliquots for ICP-MS preparation and analysis via EPA 3005A and EPA 200.8.

\footnotetext{
${ }^{1}$ PerkinElmer is a registered trademark of the Perkin Elmer Corporation, Wellesley, Massachusetts.
} 


\subsection{AMERICAN NATIONAL STANDARDS INSTITUTE LEACH PROCEDURE}

The castings were also subjected to the protocol outlined in ANSI/ANS-16.1-2003, Measurement of the Leachability of Solidified Low-Level Radioactive Wastes by a Short-Term Test Procedure.

The casting matrix for the TCLP and ANSI Leach is presented in Table 4.

Table 4. Casting Matrix for TCLP and ANSI Procedures. (2 sheets)

\begin{tabular}{|c|c|c|c|c|}
\hline Description & Material & $\begin{array}{l}\text { Units } \\
\text { (g) }\end{array}$ & $\begin{array}{c}\text { Number } \\
\text { Cast for } \\
\text { TCLP } \\
\end{array}$ & $\begin{array}{c}\begin{array}{c}\text { Number Cast } \\
\text { for }\end{array} \\
\text { ANSI/ANS 16.1 } \\
\end{array}$ \\
\hline \multirow[t]{4}{*}{ Cold blank } & Basin $43 \mathrm{GW}$ brine $-{ }^{99} \mathrm{Tc}$ & 110 & \multirow[t]{4}{*}{2} & \multirow[t]{4}{*}{2} \\
\hline & Portland cement: & 33.34 & & \\
\hline & Blast furnace slag: & 16.7047 & & \\
\hline & Getter: none & --- & & \\
\hline \multirow[t]{4}{*}{ Hot blank } & Basin $43 \mathrm{GW}$ brine $+{ }^{99} \mathrm{Tc}$ & 109.71 & \multirow[t]{4}{*}{2} & \multirow[t]{4}{*}{2} \\
\hline & Portland cement & 66.612 & & \\
\hline & Blast furnace slag & 33.37 & & \\
\hline & Getter: none & --- & & \\
\hline \multirow[t]{4}{*}{ Fishbone } & Basin $43 \mathrm{GW}$ brine $+{ }^{99} \mathrm{Tc}$ & 110 & \multirow[t]{4}{*}{2} & \multirow[t]{4}{*}{2} \\
\hline & Portland cement: & 66.63 & & \\
\hline & Blast furnace slag: & 33.29 & & \\
\hline & Getter: fishbone & 10.01 & & \\
\hline \multirow[t]{4}{*}{ Zero valent iron } & Basin $43 \mathrm{GW}$ brine $+{ }^{99} \mathrm{Tc}$ & 110.09 & \multirow[t]{4}{*}{2} & \multirow[t]{4}{*}{2} \\
\hline & Portland cement: & 66.6 & & \\
\hline & Blast furnace slag: & 33.34 & & \\
\hline & Getter: Zero valent iron & 10 & & \\
\hline \multirow[t]{4}{*}{ Bone black } & Basin $43 \mathrm{GW}$ Brine $+{ }^{99} \mathrm{Tc}$ & 110.02 & \multirow[t]{4}{*}{2} & \multirow[t]{4}{*}{2} \\
\hline & Portland cement: & 66.6 & & \\
\hline & Blast furnace slag: & 33.31 & & \\
\hline & Getter: bone black & 10.01 & & \\
\hline \multirow[t]{4}{*}{ Iron III phosphate } & Basin $43 \mathrm{GW}$ brine $+{ }^{99} \mathrm{Tc}$ & 110 & \multirow[t]{4}{*}{2} & \multirow[t]{4}{*}{2} \\
\hline & Portland cement: & 66.675 & & \\
\hline & Blast furnace slag: & 33.33 & & \\
\hline & Getter: iron III phosphate & 10 & & \\
\hline \multirow[t]{4}{*}{ Hydrotalcite } & Basin 43 GW Brine $+{ }^{99} \mathrm{Tc}$ & 66.0065 & \multirow[t]{4}{*}{2} & \multirow[t]{4}{*}{2} \\
\hline & Portland cement: & 39.99 & & \\
\hline & Blast furnace slag: & 19.99 & & \\
\hline & Getter: Hydrotalcite & 6.05 & & \\
\hline Fishbone ORP adjusted & $\begin{array}{l}\text { Basin } 43 \mathrm{GW} \text { brine }+{ }^{99} \mathrm{Tc} \\
\text { Portland cement: } \\
\text { Blast furnace slag: } \\
\text { Getter: fishbone }\end{array}$ & $\begin{array}{r}110.05 \\
66.62 \\
33.32 \\
10.02 \\
\end{array}$ & 2 & 2 \\
\hline \multirow[t]{4}{*}{ Bone black ORP adjusted } & Basin $43 \mathrm{GW}$ brine $+{ }^{99} \mathrm{Tc}$ & 110.01 & & \\
\hline & Portland cement: & 66.61 & & \\
\hline & Blast furnace slag: & 33.3 & & \\
\hline & Getter: bone black & 10.02 & & \\
\hline
\end{tabular}


RPP-PLAN-39195, Rev.0

Table 4. Casting Matrix for TCLP and ANSI Procedures. (2 sheets)

\begin{tabular}{|c|c|c|c|c|}
\hline Description & Material & $\begin{array}{c}\text { Units } \\
\text { (g) }\end{array}$ & $\begin{array}{c}\text { Number } \\
\text { Cast for } \\
\text { TCLP } \\
\end{array}$ & $\begin{array}{c}\begin{array}{c}\text { Number Cast } \\
\text { for }\end{array} \\
\text { ANSI/ANS 16.1 } \\
\end{array}$ \\
\hline \multirow[t]{4}{*}{ Zero valent iron ORP adjusted } & Basin $43 \mathrm{GW}$ brine $+{ }^{99} \mathrm{Tc}$ & \multirow{4}{*}{$\begin{array}{r}110.02 \\
66.6 \\
33.3 \\
10.01\end{array}$} & \multirow[t]{4}{*}{2} & \multirow{4}{*}{2} \\
\hline & Portland cement: & & & \\
\hline & Blast furnace slag: & & & \\
\hline & Getter: zero valent iron & & & \\
\hline \multirow[t]{4}{*}{ Iron III phosphate ORP adjusted } & Basin $43 \mathrm{GW}$ brine $+{ }^{99} \mathrm{Tc}$ & \multirow{4}{*}{$\begin{array}{r}110.01 \\
66.63 \\
33.03 \\
10.04 \\
\end{array}$} & \multirow[t]{4}{*}{2} & \multirow[t]{4}{*}{2} \\
\hline & Portland cement: & & & \\
\hline & Blast furnace slag: & & & \\
\hline & Getter: iron III phosphate & & & \\
\hline \multirow[t]{4}{*}{ Hot blank ORP adjusted } & Basin $43 \mathrm{GW}$ brine $+{ }^{99} \mathrm{Tc}$ & \multirow{4}{*}{$\begin{array}{c}110.01 \\
66.62 \\
33.31 \\
---\end{array}$} & \multirow[t]{4}{*}{2} & \multirow[t]{4}{*}{2} \\
\hline & Portland cement: & & & \\
\hline & Blast furnace slag: & & & \\
\hline & Getter: none & & & \\
\hline
\end{tabular}

The monoliths produced for the ANSI/ANS 16.1 preparation and analysis are cylindrical and on average $3.5 \mathrm{~cm}$ in height with a diameter of $2.9 \mathrm{~cm}$. The surface area (S) is $45.1 \mathrm{~cm}^{2}$. The volume of deionized water necessary for leaching the monoliths as proscribed in the method is $451 \pm 90.2 \mathrm{mls}$ as determined by

$$
V_{L}\left(\mathrm{~cm}^{3}\right) / S\left(\mathrm{~cm}_{2}\right)=10+/-0.2(\mathrm{~cm})
$$

A clean 500-mL Teflon ${ }^{2}$ jar fitted with a screened dipper and lid was used for each leaching interval. These vessels permitted the monolith to be submerged and suspended in the water column with minimal surface occlusion. To control for potential evaporation, the vessels were securely capped during each leaching interval.

At the conclusion of the 28-day cure period, the monoliths identified for ANSI/ANS 16.1 preparation and analysis were removed from the cure vessels as a solid cylinder. Every effort was made to ensure the integrity of the entire monolith. The leaching procedure as prescribed by ANSI/ANS 16.1 requires use of deionized water as the extraction medium. A sample of each leaching solution was drawn off immediately following the leach interval end time. The monolith was then resubmerged in a fresh deionized water bath.

Table 5 shows the ANSI Leach schedule (time line) for the associated analyses.

\footnotetext{
${ }^{2}$ Teflon $^{\mathbb{B}}$ is a registered trademark of I. E. du Pont de Nemours and Company, Wilmington, Delaware.
} 
RPP-PLAN-39195, Rev.0

Table 5. ANSI Leach Schedule.

\begin{tabular}{|c|c|c|c|c|c|c|}
\hline Prep Method & $\begin{array}{c}\text { Prescribed } \\
\text { Leach Time }\end{array}$ & $\begin{array}{c}\text { Leaching } \\
\text { Start Date }\end{array}$ & $\begin{array}{c}\text { Start } \\
\text { Time }\end{array}$ & $\begin{array}{c}\text { End } \\
\text { Time }\end{array}$ & $\begin{array}{c}\text { Leaching } \\
\text { End Date }\end{array}$ & Total Time \\
\hline ANSI/ANS & 2 hours & $7 / 25 / 2008$ & $12: 20$ & $14: 22$ & $7 / 25 / 2008$ & 122 minutes \\
\hline ANSI/ANS & 5 hours & $7 / 25 / 2008$ & $14: 46$ & $19: 26$ & $7 / 25 / 2008$ & 160 minutes \\
\hline ANSI/ANS & 17 hours & $7 / 26 / 2008$ & $20: 00$ & $13: 13$ & $7 / 27 / 2008$ & 1033 minutes \\
\hline ANSI/ANS & 24 hours & $7 / 27 / 2008$ & $13: 52$ & $13: 42$ & $7 / 28 / 2008$ & 1430 minutes \\
\hline ANSI/ANS & 24 hours & $7 / 28 / 2008$ & $14: 03$ & $13: 36$ & $7 / 29 / 2008$ & 1413 minutes \\
\hline ANSI/ANS & 24 hours & $7 / 29 / 2008$ & $14: 06$ & $13: 47$ & $7 / 30 / 2008$ & 1421 minutes \\
\hline ANSI/ANS & 24 hours & $7 / 30 / 2008$ & $14: 13$ & $15: 20$ & $7 / 31 / 2008$ & 1447 minutes \\
\hline & 120 hours & \multicolumn{7}{|l}{} & 7026 minutes \\
\hline
\end{tabular}

\section{RESULTS}

\subsection{DISTRIBUTION COEFFICIENT}

Table 6 shows the calculated values of the $K_{d}$ values versus the getters.

Table 6. Preliminary Results Distribution Coefficients

(ORP adjusted are highlighted).

\begin{tabular}{|l|c|c|c|}
\hline \multicolumn{1}{|c|}{ Getter } & $\mathbf{p H}$ & $\begin{array}{c}\text { ORP Adjust to - } \\
\mathbf{4 0 0} \mathbf{~ m V}\end{array}$ & $\mathbf{K}_{\mathbf{d}}$ \\
\hline Bone black & $5-6$ & No & 4.84 \\
\hline Bone black & 12 & No & $\mathrm{NA}^{*}$ \\
\hline Bone black & 12 & Yes & 6066.2 \\
\hline Fishbone & $5-6$ & No & 4.83 \\
\hline Fishbone & 12 & No & 0 \\
\hline Fishbone & 12 & Yes & 6397.6 \\
\hline Zero valent iron & $5-6$ & No & 13.9 \\
\hline Zero valent iron & 12 & No & NA \\
\hline Zero valent iron & 12 & Yes & 9202.4 \\
\hline Iron (III) phosphate & $5-6$ & No & 1.57 \\
\hline Iron (III) phosphate & 12 & No & 8.17 \\
\hline Iron (III) phosphate & 12 & Yes & 1823.3 \\
\hline
\end{tabular}

* $\mathrm{NA}=$ the sample showed more technetium after reaction with the getter, yielding a negative $\mathrm{K}_{\mathrm{d}}$ value.

Table 7 shows the getters as a percent recovery of technetium. 
Table 7. Preliminary Results Percent Recovery, (ORP adjusted are highlighted).

\begin{tabular}{|l|c|c|c|c|c|}
\hline \multicolumn{1}{|c|}{ Sample } & $\mathbf{p H}$ & $\begin{array}{c}\text { ORP Adjust to } \\
\mathbf{- 4 0 0} \mathbf{~ m V}\end{array}$ & $\begin{array}{c}\mathbf{9 9} \mathbf{T c}, \mathbf{a f t e r} \\
\mathbf{g e t t e r}(\mathbf{m g} \mathbf{L} \mathbf{)}\end{array}$ & $\begin{array}{c}\mathbf{9 9} \mathbf{T c}, \mathbf{b e f o r e} \\
\mathbf{g e t t e r}(\mathbf{m g} \mathbf{L})\end{array}$ & $\begin{array}{c}\text { Recovery } \\
\mathbf{( \% )}\end{array}$ \\
\hline Cold blank & $5-6$ & No & $<0.050$ & $<0.050$ & NA \\
\hline Cold blank & 12 & Yes & $<0.050$ & $<0.050$ & NA \\
\hline Hot blank & $5-6$ & No & 7.1 & 8.5 & 84 \\
\hline Hot blank, duplicate & $5-6$ & No & 7.1 & 8.5 & 84 \\
\hline Hot blank & 12 & No & 5.8 & 8.5 & 68 \\
\hline $\begin{array}{l}\text { Hot blank, } \\
\text { Duplicate }\end{array}$ & 12 & No & 5.8 & 8.5 & 68 \\
\hline Hot blank & 12 & Yes & 0.027 & 8.5 & 0 \\
\hline Bone black & $5-6$ & No & 6.8 & 7.1 & 96 \\
\hline Bone black & 12 & No & 7.3 & 5.8 & 126 \\
\hline Bone black & 12 & Yes & 0.10 & 5.8 & 2 \\
\hline Fishbone & $5-6$ & No & 6.8 & 7.1 & 96 \\
\hline Fishbone & 12 & No & 5.8 & 5.8 & 100 \\
\hline Fishbone & 12 & Yes & 0.096 & 5.8 & 2 \\
\hline Zero valent iron & $5-6$ & No & 6.3 & 7.1 & 89 \\
\hline Zero valent iron & 12 & No & 7 & 5.8 & 121 \\
\hline Zero valent iron & 12 & Yes & 0.068 & 5.8 & 1 \\
\hline Iron (III) phosphate & $5-6$ & No & 7 & 7.1 & 99 \\
\hline Iron (III) phosphate & 12 & No & 5.4 & 5.8 & 93 \\
\hline Iron (III) phosphate & 12 & Yes & 0.33 & 5.8 & 6 \\
\hline
\end{tabular}

\subsection{TOXICITY CHARACTERISTIC LEACHING PROCEDURE}

The initial results are presented in Table 8 .

Table 8. Preliminary Results of the TCLP (ORP adjusted are checked).

\begin{tabular}{|l|c|c|}
\hline \multicolumn{1}{|c|}{ Getter } & ORP Adjustment & $\begin{array}{c}\text { Average }{ }^{\text {99 }} \mathbf{T c ,} \\
\text { Concentration ppm }\end{array}$ \\
\hline Cold blank & & $<0.0010$ \\
\hline Hot blank & & 0.0066 \\
\hline Hot blank & $\checkmark$ & 0.0089 \\
\hline Fishbone & & 0.0072 \\
\hline Fishbone & $\checkmark$ & 0.0091 \\
\hline Zero valent iron & & 0.0029 \\
\hline Zero valent iron & $\checkmark$ & 0.0082 \\
\hline Bone black & & 0.0087 \\
\hline Bone black & $\checkmark$ & 0.0095 \\
\hline Iron III phosphate & & 0.0092 \\
\hline Iron III phosphate & $\checkmark$ & 0.0082 \\
\hline Hydrotalcite & & 0.0132 \\
\hline
\end{tabular}




\subsection{AMERICAN NATIONAL STANDARDS INSTITUTE LEACH PROCEDURE}

Table 9 gives the preliminary results of the ANSI Leach.

Table 9. ANSI/ANS 16.1 ICP-MS Preliminary Data. (3 sheets)

\begin{tabular}{|c|c|c|c|c|c|c|}
\hline Getter ID & Lab ID & $\begin{array}{c}\text { ORP } \\
\text { Adjusted } \\
\text { by Iron II } \\
\text { Sulfate }\end{array}$ & $\begin{array}{c}\text { Cumulative } \\
\text { Leaching } \\
\text { Time }\end{array}$ & Analyte & $\begin{array}{l}\text { Result } \\
(\mathrm{mg} / \mathrm{L})\end{array}$ & $\begin{array}{c}\text { Reportin } \\
\text { g Limit } \\
\text { (mg/L) }\end{array}$ \\
\hline Cold blank & WA310720080031-001 & No & 2 hours & ${ }^{99} \mathrm{Tc}^{7+}$ & $<0.0010$ & 0.0010 \\
\hline Cold blank & WA310720080032-001 & No & 5 hours & ${ }^{99} \mathrm{Tc}^{7+}$ & $<0.0010$ & 0.0010 \\
\hline Cold blank & WA010820080014-001 & No & 17 hours & ${ }^{99} \mathrm{Tc}^{7+}$ & $<0.0010$ & 0.0010 \\
\hline Cold blank & WA010820080018-001 & $\overline{\text { No }}$ & 48 hours & ${ }^{99} \mathrm{Tc}^{7+}$ & $<0.0010$ & 0.0010 \\
\hline Cold blank & WA010820080002-001 & No & 72 hours & ${ }^{99} \mathrm{Tc}^{7+}$ & $<0.0010$ & 0.0010 \\
\hline Cold blank & WA010820080018-001 & No & 96 hours & ${ }^{99} \mathrm{Tc}^{7+}$ & $<0.0010$ & 0.0010 \\
\hline Cold blank & WA310720080031-001 & No & 120 hours & ${ }^{99} \mathrm{Tc}^{7+}$ & $<0.0010$ & 0.0010 \\
\hline Hot blank & WA310722080031-003 & No & 2 hours & ${ }^{99} \mathrm{Tc}^{7+}$ & $<0.0010$ & 0.0010 \\
\hline $\begin{array}{l}\text { Hot blank } \\
\text { (average) }\end{array}$ & WA310720080031-022 & Yes & 2 hours & ${ }^{99} \mathrm{Tc}^{7+}$ & 0.0010 & 0.0010 \\
\hline Hot bank & WA310722080032-003 & No & 5 hours & ${ }^{99} \mathrm{Tc}^{7+}$ & $<0.0010$ & 0.0010 \\
\hline Hot blank & WA310720080032-022 & Yes & 5 hours & ${ }^{99} \mathrm{Tc}^{7+}$ & $<0.0010$ & 0.0010 \\
\hline Hot blank & WA010820080014-003 & No & 17 hours & ${ }^{99} \mathrm{Tc}^{7+}$ & $<0.0010$ & 0.0010 \\
\hline Hot blank & WA010820080014-022 & Yes & 17 hours & ${ }^{99} \mathrm{Tc}^{7+}$ & $<0.0010$ & 0.0010 \\
\hline Hot blank & WA010820080001-003 & No & 48 hours & ${ }^{99} \mathrm{Tc}^{7+}$ & $<0.0010$ & 0.0010 \\
\hline Hot blank & WA010820080001-022 & Yes & 48 hours & ${ }^{99} \mathrm{Tc}^{7+}$ & $<0.0010$ & 0.0010 \\
\hline Hot blank & WA010820080002-003 & No & 72 hours & ${ }^{99} \mathrm{Tc}^{7+}$ & $<0.0010$ & 0.0010 \\
\hline Hot blank & WA010820080002-022 & Yes & 72 hours & ${ }^{99} \mathrm{Tc}^{7+}$ & $<0.0010$ & 0.0010 \\
\hline Hot blank & WA010820080018-003 & No & 96 hours & ${ }^{99} \mathrm{Tc}^{7+}$ & $<0.0010$ & 0.0010 \\
\hline Hot blank & WA010820080018-022 & Yes & 96 hours & ${ }^{99} \mathrm{Tc}^{7+}$ & $<0.0010$ & 0.0010 \\
\hline Hot blank & WA310722080031-003 & No & 120 hours & ${ }^{99} \mathrm{Tc}^{7+}$ & $<0.0010$ & 0.0010 \\
\hline Hot blank & WA310722080031-022 & Yes & 120 hours & ${ }^{99} \mathrm{Tc}^{7+}$ & $<0.0010$ & 0.001 \\
\hline Bone black & WA310720080031-009 & No & 2 hours & ${ }^{99} \mathrm{Tc}^{7+}$ & $<0.0010$ & 0.0010 \\
\hline Bone black & WA310720080031-016 & Yes & 2 hours & ${ }^{99} \mathrm{Tc}^{7+}$ & $<0.0010$ & 0.0010 \\
\hline Bone black & WA310720080032-009 & No & 5 hours & ${ }^{99} \mathrm{Tc}^{7+}$ & $<0.0010$ & 0.0010 \\
\hline Bone black & WA310720080032-016 & Yes & 5 hours & ${ }^{99} \mathrm{Tc}^{7+}$ & $<0.0010$ & 0.0010 \\
\hline Bone black & WA010820080014-009 & No & 17 hours & ${ }^{99} \mathrm{Tc}^{7+}$ & $<0.0010$ & 0.0010 \\
\hline Bone black & WA010820080014-016 & Yes & 17 hours & ${ }^{99} \mathrm{Tc}^{7+}$ & $<0.0010$ & 0.0010 \\
\hline Bone black & WA010820080001-009 & No & 48 hours & ${ }^{99} \mathrm{Tc}^{7+}$ & $<0.0010$ & 0.0010 \\
\hline Bone black & WA010820080001-016 & Yes & 48 hours & ${ }^{99} \mathrm{Tc}^{7+}$ & $<0.0010$ & 0.0010 \\
\hline Bone black & WA010820080002-016 & Yes & 72 hours & ${ }^{99} \mathrm{Tc}^{7+}$ & $<0.0010$ & 0.0010 \\
\hline $\begin{array}{l}\text { Bone black } \\
\text { (average) }\end{array}$ & WA010820080002-009 & No & 72 hours & ${ }^{99} \mathrm{Tc}^{7+}$ & 0.008 & 0.0010 \\
\hline Bone black & WA010820080018-009 & No & 96 hours & ${ }^{99} \mathrm{Tc}^{7+}$ & $<0.0010$ & 0.0010 \\
\hline Bone black & WA010820080018-016 & Yes & 96 hours & ${ }^{99} \mathrm{Tc}^{7+}$ & $<0.0010$ & 0.0010 \\
\hline
\end{tabular}


RPP-PLAN-39195, Rev.0

Table 9. ANSI/ANS 16.1 ICP-MS Preliminary Data. (3 sheets)

\begin{tabular}{|c|c|c|c|c|c|c|}
\hline Getter ID & Lab ID & $\begin{array}{c}\text { ORP } \\
\text { Adjusted } \\
\text { by Iron II } \\
\text { Sulfate }\end{array}$ & $\begin{array}{c}\text { Cumulative } \\
\text { Leaching } \\
\text { Time }\end{array}$ & Analyte & $\begin{array}{l}\text { Result } \\
(\mathrm{mg} / \mathrm{L})\end{array}$ & $\begin{array}{c}\text { Reportin } \\
\text { g Limit } \\
(\mathrm{mg} / \mathrm{L})\end{array}$ \\
\hline Bone black & WA310722080031-009 & No & 120 hours & ${ }^{99} \mathrm{Tc}^{7+}$ & $<0.0010$ & 0.0010 \\
\hline Bone black & WA310722080031-016 & Yes & 120 hours & ${ }^{99} \mathrm{Tc}^{7+}$ & $<0.0010$ & 0.0010 \\
\hline Fishbone & WA310720080031-005 & No & 2 hours & ${ }^{99} \mathrm{Tc}^{7+}$ & $<0.0010$ & 0.0010 \\
\hline Fishbone & WA310720080031-014 & Yes & 2 hours & ${ }^{99} \mathrm{Tc}^{7+}$ & $<0.0010$ & 0.0010 \\
\hline Fishbone & WA310720080032-007 & No & 5 hours & ${ }^{99} \mathrm{Tc}^{7+}$ & $<0.0010$ & 0.0010 \\
\hline Fishbone & WA310720080032-014 & Yes & 5 hours & ${ }^{99} \mathrm{Tc}^{7+}$ & $<0.0010$ & 0.0010 \\
\hline Fishbone & WA010820080002-005 & No & 17 hours & ${ }^{99} \mathrm{Tc}^{7+}$ & $<0.0010$ & 0.0010 \\
\hline Fishbone & WA010820080014-014 & Yes & 17 hours & ${ }^{99} \mathrm{Tc}^{7+}$ & $<0.0010$ & 0.0010 \\
\hline Fishbone & WA010820080001-005 & No & 48 hours & ${ }^{99} \mathrm{Tc}^{7+}$ & $<0.0010$ & 0.0010 \\
\hline Fishbone & WA010820080001-014 & Yes & 48 hours & ${ }^{99} \mathrm{Tc}^{7+}$ & $<0.0010$ & 0.0010 \\
\hline Fishbone & WA010820080002-005 & No & 72 hours & ${ }^{99} \mathrm{Tc}^{7+}$ & $<0.0010$ & 0.0010 \\
\hline Fishbone & WA010820080002-014 & Yes & 72 hours & ${ }^{99} \mathrm{Tc}^{7+}$ & $<0.0010$ & 0.0010 \\
\hline Fishbone & WA010820080018-005 & No & 96 hours & ${ }^{99} \mathrm{Tc}^{7+}$ & $<0.0010$ & 0.0010 \\
\hline Fishbone & WA010820080018-014 & Yes & 96 hours & ${ }^{99} \mathrm{Tc}^{7+}$ & $<0.0010$ & 0.0010 \\
\hline Fishbone & WA310722080031-005 & No & 120 hours & ${ }^{99} \mathrm{Tc}^{7+}$ & $<0.0010$ & 0.0010 \\
\hline Fishbone & WA310722080031-014 & Yes & 120 hours & ${ }^{99} \mathrm{Tc}^{7+}$ & $<0.0010$ & 0.0010 \\
\hline Hydrotalcite & WA310720080031-013 & No & 2 hours & ${ }^{99} \mathrm{Tc}^{7+}$ & $<0.0010$ & 0.0010 \\
\hline Hydrotalcite & WA310720080032-013 & No & 5 hours & ${ }^{99} \mathrm{Tc}^{7+}$ & $<0.0010$ & 0.0010 \\
\hline Hydrotalcite & WA010820080014-013 & No & 17 hours & ${ }^{99} \mathrm{Tc}^{7+}$ & $<0.0010$ & 0.0010 \\
\hline Hydrotalcite & WA010820080001-013 & No & 48 hours & ${ }^{99} \mathrm{Tc}^{7+}$ & $<0.0010$ & 0.0010 \\
\hline Hydrotalcite & WA010820080002-013 & No & 72 hours & ${ }^{99} \mathrm{Tc}^{7+}$ & $<0.0010$ & 0.0010 \\
\hline Hydrotalcite & WA010820080018-013 & No & 96 hours & ${ }^{99} \mathrm{Tc}^{7+}$ & $<0.0010$ & 0.0010 \\
\hline Hydrotalcite & WA310722080031-013 & No & 120 hours & ${ }^{99} \mathrm{Tc}^{7+}$ & $<0.0010$ & 0.0010 \\
\hline $\begin{array}{l}\text { Iron (III) } \\
\text { phosphate }\end{array}$ & WA310720080031-011 & No & 2 hours & ${ }^{99} \mathrm{Tc}^{7+}$ & $<0.0010$ & 0.0010 \\
\hline $\begin{array}{l}\text { Iron (III) } \\
\text { phosphate }\end{array}$ & WA310720080031-020 & Yes & 2 hours & ${ }^{99} \mathrm{Tc}^{7+}$ & $<0.0010$ & 0.0010 \\
\hline $\begin{array}{l}\text { Iron (III) } \\
\text { phosphate }\end{array}$ & WA310720080032-011 & No & 5 hours & ${ }^{99} \mathrm{Tc}^{7+}$ & $<0.0010$ & 0.0010 \\
\hline $\begin{array}{l}\text { Iron (III) } \\
\text { phosphate }\end{array}$ & WA310720080032-020 & Yes & 5 hours & ${ }^{99} \mathrm{Tc}^{7+}$ & $<0.0010$ & 0.0010 \\
\hline $\begin{array}{l}\text { Iron (III) } \\
\text { phosphate }\end{array}$ & WA010820080014-011 & No & 17 hours & ${ }^{99} \mathrm{Tc}^{7+}$ & $<0.0010$ & 0.0010 \\
\hline $\begin{array}{l}\text { Iron (III) } \\
\text { phosphate }\end{array}$ & WA010820080014-020 & Yes & 17 hours & ${ }^{99} \mathrm{Tc}^{7+}$ & $<0.0010$ & 0.0010 \\
\hline $\begin{array}{l}\text { Iron (III) } \\
\text { phosphate }\end{array}$ & WA010820080001-011 & No & 48 hours & ${ }^{99} \mathrm{Tc}^{7+}$ & $<0.0010$ & 0.0010 \\
\hline $\begin{array}{l}\text { Iron (III) } \\
\text { phosphate }\end{array}$ & WA010820080001-020 & Yes & 48 hours & ${ }^{99} \mathrm{Tc}^{7+}$ & $<0.0010$ & 0.0010 \\
\hline $\begin{array}{l}\text { Iron (III) } \\
\text { phosphate }\end{array}$ & WA010820080002-011 & No & 72 hours & ${ }^{99} \mathrm{Tc}^{7+}$ & $<0.0010$ & 0.0010 \\
\hline
\end{tabular}


RPP-PLAN-39195, Rev.0

Table 9. ANSI/ANS 16.1 ICP-MS Preliminary Data. (3 sheets)

\begin{tabular}{|c|c|c|c|c|c|c|}
\hline Getter ID & Lab ID & $\begin{array}{c}\text { ORP } \\
\text { Adjusted } \\
\text { by Iron II } \\
\text { Sulfate }\end{array}$ & $\begin{array}{c}\text { Cumulative } \\
\text { Leaching } \\
\text { Time }\end{array}$ & Analyte & $\begin{array}{l}\text { Result } \\
\text { (mg/L) }\end{array}$ & $\begin{array}{c}\text { Reportin } \\
\text { g Limit } \\
\text { (mg/L) }\end{array}$ \\
\hline $\begin{array}{l}\text { Iron (III) } \\
\text { phosphate }\end{array}$ & WA010820080002-020 & Yes & 72 hours & ${ }^{99} \mathrm{Tc}^{7+}$ & $<0.0010$ & 0.0010 \\
\hline $\begin{array}{l}\text { Iron (III) } \\
\text { phosphate }\end{array}$ & WA010820080018-011 & No & 96 hours & ${ }^{99} \mathrm{Tc}^{7+}$ & $<0.0010$ & 0.0010 \\
\hline $\begin{array}{l}\text { Iron (III) } \\
\text { phosphate }\end{array}$ & WA010820080018-020 & Yes & 96 hours & ${ }^{99} \mathrm{Tc}^{7+}$ & $<0.0010$ & 0.0010 \\
\hline $\begin{array}{l}\text { Iron (III) } \\
\text { phosphate }\end{array}$ & WA310722080031-011 & No & 120 hours & ${ }^{99} \mathrm{Tc}^{7+}$ & $<0.0010$ & 0.0010 \\
\hline $\begin{array}{l}\text { Iron (III) } \\
\text { phosphate }\end{array}$ & WA310722080031-020 & Yes & 120 hours & ${ }^{99} \mathrm{Tc}^{7+}$ & $<0.0010$ & 0.0010 \\
\hline Zero valent iron & WA310720080031-007 & No & 2 hours & ${ }^{99} \mathrm{Tc}^{3+}$ & $<0.0010$ & 0.0010 \\
\hline Zero valent iron & WA310720080031-018 & Yes & 2 hours & ${ }^{99} \mathrm{Tc}^{7+}$ & $<0.0010$ & 0.0010 \\
\hline Zero valent iron & WA310720080032-018 & $\overline{\text { No }}$ & 5 hours & ${ }^{99} \mathrm{Tc}^{7+}$ & $<0.0010$ & 0.0010 \\
\hline Zero valent iron & WA310720080032-019 & Yes & 5 hours & ${ }^{99} \mathrm{Tc}^{7+}$ & $<0.0010$ & 0.0010 \\
\hline Zero valent iron & WA010820080014-007 & No & 17 hours & ${ }^{99} \mathrm{Tc}^{7+}$ & $<0.0010$ & 0.0010 \\
\hline Zero valent iron & WA010820080014-018 & Yes & 17 hours & ${ }^{99} \mathrm{Tc}^{7+}$ & $<0.0010$ & 0.0010 \\
\hline Zero valent iron & WA010820080001-007 & No & 48 hours & ${ }^{99} \mathrm{Tc}^{7+}$ & $<0.0010$ & 0.0010 \\
\hline Zero valent iron & WA010820080001-018 & Yes & 48 hours & ${ }^{99} \mathrm{Tc}^{7+}$ & $<0.0010$ & 0.0010 \\
\hline Zero valent iron & WA010820080002-007 & No & 72 hours & ${ }^{99} \mathrm{Tc}^{7+}$ & $<0.0010$ & 0.0010 \\
\hline $\begin{array}{l}\text { Zero valent iron } \\
\text { (Average) }\end{array}$ & WA010820080002-018 & Yes & 72 hours & ${ }^{99} \mathrm{Tc}^{7+}$ & 0.0022 & 0.0010 \\
\hline Zero valent iron & WA010820080018-007 & No & 96 hours & ${ }^{99} \mathrm{Tc}^{7+}$ & $<0.0010$ & 0.0010 \\
\hline Zero valent iron & WA010820080018-018 & Yes & 96 hours & ${ }^{99} \mathrm{Tc}^{7+}$ & $<0.0010$ & 0.0010 \\
\hline Zero valent iron & WA310722080031-007 & No & 120 hours & ${ }^{99} \mathrm{Tc}^{7+}$ & $<0.0010$ & 0.0010 \\
\hline Zero valent iron & WA310722080031-018 & Yes & 120 hours & ${ }^{99} \mathrm{Tc}^{7+}$ & $<0.0010$ & 0.0010 \\
\hline
\end{tabular}

\section{CONCLUSIONS}

With the data in the preliminary stages, it is difficult to converge on conclusions. However, it is probably safe to conclude that the ORP adjustment to $-400 \mathrm{mV}$ versus standard hydrogen electrode does effectively reduce technetium from the +7 oxidation state to the +4 state. The +4 state is one in which the technetium oxide $\left(\mathrm{TcO}_{4}\right)$ is stable and exhibits a black precipitate (Atlas of Electrochemical Equilibria in Aqueous Solutions, Pourbaix 1974).

It is also concluded that the candidate getters would be effective in dilute solutions of pertechnetate and were not that effective in high salinity solutions, with the exception of the addition of ferrous sulfate to obtain a solution ORP of $-400 \mathrm{mV}$. There appears to be two effects exhibited by the high salinity solution. One is based on salinity itself indicating a percent reduction of technetium (Table 7). Table 7 also indicates the effect of using ferrous sulfate to adjust the solution ORP to achieve a reduced oxidation state. 
Once data is finalized, a revision of this report will be issued. It is expected that the conclusion section of the revision will be significantly expanded.

\section{REFERENCES}

ANSI/ANS-16.1-2003, Measurement of the Leachability of Solidified Low-Level Radioactive Wastes by a Short-term Test Procedure, American Nuclear Society, La Grange Park, Illinois.

CLS-RPT-816, 2008, Draft Report: Assessment of Technetium Leachability in Cement Stabilized Basin 43 Groundwater Brine, RJ Lee Group, Inc., Center for Laboratory Sciences, Pasco, Washington.

RPP-PLAN-33338, 2008, Test Plan for the Assessment of Technetium Leachability in CementStabilized Basin 43 Groundwater Brine, Rev. 1A, CH2M HILL Hanford Group, Inc., Richland, Washington

RPP-RPT-26742, 2005, Hanford Containerized Cast Stone Facility Task - 1 Process Testing and Development Final Test Report, Rev. 0, CH2M HILL Hanford Group, Inc., Richland, Washington.

RPP-RPT-31740, 2006, Development and Testing of a Cement Based Solid Waste Form Using Synthetic UP-1 Groundwater, Rev. 0, CH2M HILL Hanford Group, Inc., Richland, Washington.

SW-846, Method 1311, "Toxicity Characteristic Leaching Procedure," Test Methods for Evaluating Solid Waste, Physical/Chemical Methods, Third Edition, as amended, U.S. Environmental Protection Agency, Washington, D.C., September 1986.

Pourbaix, M., 1974, Atlas of Electrochemical Equilibria in Aqueous Solutions, National Association of Corrosion Engineers, Houston, Texas. 